\title{
Aerothermodynamic Characteristics of Flat-Nose Power-Law Bodies in Low-Density Hypersonic Flow
}

\author{
Wilson F. N. Santos* \\ National Institute for Space Research \\ Cachoeira Paulista, SP 12630-000 BRAZIL
}

\begin{abstract}
The steady-state aerodynamic characteristics of a new family of blunted leading edges immersed in high-speed rarefied air flow are examined by using a Direct Simulation Monte Carlo Method. A very detailed description of the aerodynamic surface quantities on the bodies has been presented by a numerical method that properly accounts for nonequilibrium effects that arise near the nose of the leading edges and that are especially important at high Mach number. Comparisons based on geometry are made between these blunt configurations and circular cylinder shapes. Some significant differences between these shapes is noted on the aerodynamic surface quantities. It is found that the heat transfer coefficient at the stagnation point decreased with increasing the flat-nose of the leading edges. The analysis also showed that, despite the seeming advantages of the new blunt shapes, circular cylinder still provides smaller stagnation point heating, however large total drag under the range of condition investigated.
\end{abstract}

\section{Nomenclature}

a Constant in power-law body equation, Eq.(1)

$C_{d} \quad$ Drag coefficient, $2 D / \rho_{\infty} V_{\infty}^{2} H$

$C_{f} \quad$ Skin friction coefficient, $2 \tau_{w} / \rho_{\infty} V_{\infty}^{2}$

$C_{h} \quad$ Heat transfer coefficient, $2 q_{w} / \rho_{\infty} V_{\infty}^{3}$

$C_{p} \quad$ Pressure coefficient, $2\left(p_{w}-p_{\infty}\right) / \rho_{\infty} V_{\infty}^{2}$

$D \quad$ Drag force, $\mathrm{N}$

d Molecular diameter, $\mathrm{m}$

$H \quad$ Body height at the base, $m$

$K n$ Knudsen number, $\lambda / l$

$L \quad$ Body length, m

$l \quad$ Characteristic length, $\mathrm{m}$

$M \quad$ Mach number

$m \quad$ Molecular mass, $\mathrm{kg}$

$N \quad$ Number flux, $\mathrm{m}^{-2} s^{-1}$

$n \quad$ Body power law exponent

$p$ Pressure, $\mathrm{N} / \mathrm{m}^{2}$

$q \quad$ Heat flux, $\mathrm{W} / \mathrm{m}^{2}$

$R \quad$ Circular cylinder radius, $\mathrm{m}$

Re Reynolds number, $\rho V l / \mu$

$s \quad$ Arc length, $\mathrm{m}$

${ }^{*}$ Researcher, Combustion and Propulsion Laboratory. AIAA Member. 
$t \quad$ Leading edge thickness, $\mathrm{m}$

$V \quad$ Velocity, $\mathrm{m} / \mathrm{s}$

$X$ Mole fraction

$x, y \quad$ Cartesian axes in physical space, $\mathrm{m}$

$y_{\text {nose }}$ Half thickness of the leading edge, $\mathrm{m}$

$\eta \quad$ Coordinate normal to body surface, $\mathrm{m}$

$\theta \quad$ Wedge half angle, deg

$\lambda \quad$ Mean free path, $\mathrm{m}$

$\xi \quad$ Coordinate tangent to body surface, $\mathrm{m}$

$\rho \quad$ Density, $\mathrm{kg} / \mathrm{m}^{3}$

$\tau \quad$ Shear stress, $\mathrm{N} / \mathrm{m}^{2}$

$\omega \quad$ Viscosity index

\section{Subscript}

cyl Circular cylinder

$o \quad$ Stagnation point conditions

pwr Power law

$w \quad$ Wall conditions

$\infty \quad$ Freestream conditions

\section{Introduction}

O $\mathrm{NE}$ of the key issues concerning hypersonic configurations is the leading edge of the vehicle. Hypersonic configurations are generally characterized by slender bodies and sharp leading edges in order to achieve good aerodynamic properties like high lift and low drag. Nevertheless, for flight at hypersonic speeds, the vehicle leading edges must be sufficiently blunt in order to reduce the heat transfer rate to acceptable levels, and possibly to allow for internal heat conduction. The use of blunt-nose shapes tends to alleviate the aerodynamic heating problem since the heat flux for blunt bodies is far lower than that for sharply pointed bodies. In addition, the reduction in heating rate for a blunt body is accompanied by an increase in heat capacity, due to the increased volume. Therefore, designing a hypersonic vehicle leading edge involves a tradeoff between making the leading edge sharp enough to obtain acceptable aerodynamic and propulsion efficiency and blunt enough to reduce the aerodynamic heating in the stagnation point. In this context, power law shaped leading edges $\left(y \propto x^{n}, 0<n<1\right)$ have been considered as especially promising bluntness for hypersonic configurations in order to provide the leading edge heating and manufacturing requirements. This concept is based on the work of Mason and Lee ${ }^{1}$, who have pointed out that, for certain exponents, power law shapes exhibit both blunt (infinite body slope at the nose) and sharp (zero radius of curvature at the nose) characteristics. They suggested the possibility of a difference between shapes that are geometrically sharp and shapes that behave aerodynamically as if they were sharp. Their analysis describes the details of the geometry and aerodynamics of low-drag axisymmetric bodies by using Newtonian theory. However, one of the important aspects of the problem, stagnation point heat transfer, was not considered.

A great deal of works ${ }^{2-9}$ has been carried out recently on power-law form representing blunt geometries. The major interest in these works has gone into considering the power-law shape as possible candidates for blunting geometries of hypersonic leading edges, such as hypersonic waverider vehicles ${ }^{10}$ which have been lately considered for high-altitude/low-density applications ${ }^{11-14}$.

Through the use of Direct Simulation Monte Carlo (DSMC) method, Santos and Lewis ${ }^{4}$ have investigated the sensitivity of the stagnation point heating and total drag to shape variations of such leading edges for the idealized situation of two-dimensional rarefied hypersonic flow at zero angle of incidence. The emphasis of the work was to compare power law leading edges with round leading edges (circular cylinder) in order to determine which geometry would be better suited as a blunting profile in terms of stagnation point heating 
and total drag coefficient. Their analysis showed that power law leading edges provided smaller total drag and larger stagnation point heating than the corresponding circular cylinder that generated the power law shapes under the range of conditions investigated.

\section{Motivation}

In the present work a new investigation of a stagnation point heating reduction concept for hypersonic flow on power law shapes is conducted. According to Reller ${ }^{15}$, a method of designing low heat transfer bodies is devised on the premise that the rate of heat transfer to the nose will be low if the local velocity is low, while the rate of heat transfer to the afterbody will be low if the local density is low. A typical body that results from this design method consists of a flat nose followed by a highly curved, but for the most part slightly inclined, afterbody surface. In this scenario, the purpose of this work is to examine computationally the aerodynamic surface quantities for a family of new contours, flat-nose power-law shapes, that satisfy these geometric constraints and to compare them to round leading edges in order to provide information on how well these shapes stand up as possible candidates for blunting geometries of hypersonic leading edges. The focus of the present study is the low-density region in the upper atmosphere. At high altitudes, and therefore low density, the molecular collision rate is low and the energy exchange occurs under nonequilibrium conditions. In such a circumstance, the conventional continuum gas dynamics that are based on the concept of local equilibrium is inappropriate, and an approach based on molecular gas dynamics is required for the analysis. Therefore, a DSMC method will be employed to calculate the rarefied hypersonic two-dimensional flow on the flat-nose leading edge shapes.

\section{Leading Edge Geometry Definition}

In dimensional form, the power law contours that define the shapes of the afterbody surfaces are given by the following expression,

$$
y=y_{\text {nose }}+a x^{n}
$$

where $y_{n o s e}$ is the half thickness of the flat nose of the leading edges, $n$ is the power law exponent and $a$ is the power law constant which is a function of $n$.

The flat-nose power-law shapes are modeled by assuming a sharp leading edge (wedge) of half angle $\theta$ with a circular cylinder of radius $R$ inscribed tangent to this wedge. The flat-nose power law shapes, inscribed between the wedge and the cylinder, are also tangent to both shapes at the same common point where they have the same slope angle. It was assumed a leading edge half angle of 10 degree, a circular cylinder diameter of $10^{-2} \mathrm{~m}$, power law exponents of $2 / 3,0.7,3 / 4$, and 0.8 , and front surface thicknesses $t / \lambda_{\infty}$ of $0,0.01,0.1$ and 1 , where $t=2 y_{\text {nose }}$ and $\lambda_{\infty}$ is the freestream molecular mean free path. Figure 1 shows schematically this con-

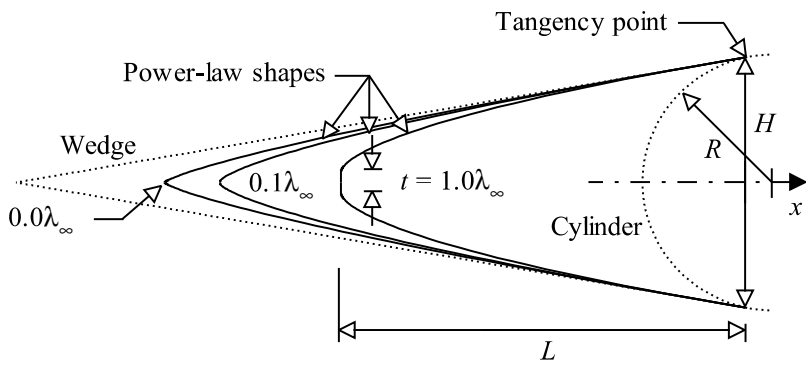

Figure 1. Drawing illustrating the leading edge geometries. struction.

From geometric considerations, the power law constant $a$ is obtained by matching slopes for the wedge, circular cylinder and power law body at the tangency point. The common body height $H$ at the tangency point is equal to $2 R \cos \theta$, and the body length $L$ from the nose to the tangency point in the axis of symmetry is given by $n(H-t) / 2 \tan \theta$. Since the wake region behind the power law bodies is not of interest in this investigation, it was assumed that the power law bodies are infinitely long but only the length $L$ is considered. 


\section{Methodology}

The various phenomena found in high-speed flow are often described in terms of dimensionless parameters of the flow such as Reynolds number and Mach number. The former parameter may be considered to be a measure of the effect of viscosity and the latter parameter a measure of the effect of compressibility on the flowfield. An additional parameter, the Knudsen number, becomes important when considering rarefied flow. The Knudsen number, $K n=\lambda / l$, is defined as the ratio of the mean free path $\lambda$ of the molecules to a characteristic body dimension $l$. Traditionally, flows are divided into four regimes ${ }^{16}: K n<0.01$, continuum flow, $0.01<K n<0.1$, slip flow, $0.1<K n<10$, transitional flow, and $K n>10$, free molecular flow.

Flow in the regime of intermediate Knudsen numbers, $0.01<K n<10$, is difficult to deal with analytically and, at this time, it appears that the Direct Simulation Monte Carlo (DSMC) method introduced by Bird ${ }^{17}$ is the most accurate and credible procedure for computing leading edge flows and surface effects in this flow regime. The DSMC method models the flow as being a large number of statistically representative particles, each one with a position, velocity and internal energy. The state of the particles is stored and modified with time as the particles move, collide, and undergo boundary interactions in simulated physical space.

The molecular collisions are modeled by using the variable hard sphere (VHS) molecular model ${ }^{18}$ and the no time counter (NTC) collision sampling technique ${ }^{19}$. The VHS model employs the simple hard sphere angular scattering law so that all directions are equally possible for post-collision velocity in the centerof-mass frame of reference. However, the collision cross section depends on the relative speed of colliding molecules. The intermolecular collisions are uncoupled to the translational molecular motion over the time step used to advance the simulation. Time is advanced in discrete steps such that each step is small in comparison with the mean collision time ${ }^{20,21}$. The simulation is always calculated as unsteady flow. However, a steady flow solution is obtained as the large time state of the simulation.

The energy exchange between kinetic and internal modes is controlled by the Borgnakke-Larsen statistical model $^{22}$. The essential feature of this model is that a part of collisions is treated as completely inelastic, and the remainder of the molecular collisions is regarded as elastic. Simulations are performed using a non-reacting gas model consisting of two chemical species, $\mathrm{N}_{2}$ and $\mathrm{O}_{2}$. Energy exchanges between the translational and internal modes are considered. The probability of an inelastic collision determines the rate at which energy is transferred between the translational and internal modes after an inelastic collision. For a given collision, the probabilities are designated by the inverse of the relaxation numbers, which correspond to the number of collisions necessary, on average, for a molecule to relax. The relaxation numbers are traditionally given as constants, 5 for rotation and 50 for vibration.

In order to implement the particle-particle collisions, the flowfield is divided into an arbitrary number of regions, which are subdivided into computational cells. The cells are further subdivided into 4 subcells, 2 subcells/cell in each direction. The cell provides a convenient reference sampling of the macroscopic gas properties, while the collision partners are selected from the same subcell for the establishment of the collision rate. The dimensions of the cells must be such that the change in flow properties across each cell is small. The linear dimensions of the cells should be small in comparison with the scale length of the macroscopic flow gradients normal to streamwise directions, which means that the cell dimensions should be of the order of the local mean free path or even smaller ${ }^{23,24}$.

The computational domain used for the calculation is made large enough so that body disturbances do not reach the upstream and side boundaries, where freestream conditions are specified. A schematic view of the computational domain is depicted in Fig. 2. Side I is defined by the body surface. Diffuse reflection with complete thermal accommodation is the condition applied to this side. Advantage of the flow symmetry is taken into account, and molecular simulation is applied to one-half of a full configuration. Thus, side II is a plane of symmetry. In such a boundary, all flow gradients normal to the plane are zero. At the molecular level, this plane is equivalent to a specular reflecting boundary. Side III is the freestream side through which simulated molecules enter and exit. Finally, the flow at the downstream outflow boundary, side IV, is predominantly supersonic and vacuum condition is specified ${ }^{17}$. At this boundary, simulated molecules can only exit.

4 of 14 
Numerical accuracy in DSMC method depends on the grid resolution chosen as well as the number of particles per computational cell. Both effects were investigated to determine the number of cells and the number of particles required to achieve grid independence solutions for the thermal non-equilibrium flow that arises near the nose of the leading edges. Grid independence was tested by running the calculations with half and double the number of cells in each direction compared to a standard grid. Solutions were near identical for all grids used and were considered fully grid independent. A discussion of both effects on the aerodynamic surface quantities for power law shapes with zero-thickness nose $\left(t / \lambda_{\infty}\right.$ $=0)$ is described in details in Santos and Lewis ${ }^{8}$. The same procedure was adopted for the $t / \lambda_{\infty}>0$ cases. However, the discussion will not be presented here.

\section{Flow Conditions}

The freestream flow conditions used for the numerical simulation of flow past the leading edges are those given by Santos and Lewis ${ }^{4}$ and summarized in Table 1, and the gas properties ${ }^{17}$ are shown in Table 2.

The freestream velocity $V_{\infty}$ is assumed to be constant at $3.5 \mathrm{~km} / \mathrm{s}$, which corresponds to a freestream Mach number $M_{\infty}$ of 12 . The translational and vibrational temperatures in the freestream are in equilibrium at $220 \mathrm{~K}$, and the leading edge surface has a constant wall temperature $T_{w}$ of $880 \mathrm{~K}$ for all cases considered. The freestream Reynolds number by unit meter $R e_{\infty}$ is 21455 based on conditions in the undisturbed stream.

The overall Knudsen number $K n_{t}$, defined as $\lambda_{\infty} / t$, corresponds to $\infty, 100,10$ and 1 for thickness, $t / \lambda_{\infty}$ of $0,0.01,0.1$ and 1 , respectively. It is important to mention that $K n_{t}=\infty$ case corresponds to the power law leading edge set already investigated by Santos and Lewis ${ }^{4}$.

Table 1. Freestream flow conditions

\begin{tabular}{ccccccc}
\hline \hline Altitude $(\mathrm{km})$ & $T_{\infty}(\mathrm{K})$ & $p_{\infty}\left(\mathrm{N} / \mathrm{m}^{2}\right)$ & $\rho_{\infty}\left(\mathrm{kg} / \mathrm{m}^{3}\right)$ & $\mu_{\infty}\left(\mathrm{Ns} / \mathrm{m}^{2}\right)$ & $n_{\infty}\left(\mathrm{m}^{-3}\right)$ & $\lambda_{\infty}(\mathrm{m})$ \\
\hline 70 & 220.0 & 5.582 & $8.753 \times 10^{-2}$ & $1.455 \times 10^{-2}$ & $1.8209 \times 10^{21}$ & $9.03 \times 10^{-4}$ \\
\hline \hline
\end{tabular}

Table 2. Gas properties

\begin{tabular}{ccccc}
\hline \hline & $X$ & $m(\mathrm{~kg})$ & $d(\mathrm{~m})$ & $\omega$ \\
\hline $\mathrm{O}_{2}$ & 0.237 & $5.312 \times 10^{-26}$ & $4.01 \times 10^{-10}$ & 0.77 \\
$N_{2}$ & 0.763 & $4.650 \times 10^{-26}$ & $4.11 \times 10^{-10}$ & 0.74 \\
\hline \hline
\end{tabular}




\section{Computational Results and Discussions}

Attention is now focused on the calculations of the aerodynamic surface quantities obtained from the DSMC results. Aerodynamic surface quantities of particular interest are number flux, heat transfer, pressure, skin friction and drag. Therefore, the purpose of this section is to discuss differences in these properties due to variations on the nose thickness of the power law bodies and to compare them to those obtained for the reference circular cylinder that generated the power law shapes. Comparisons based on geometry are made to examine the benefits and disadvantages of using these blunt geometries over circular cylinders.

\section{A. Number Flux}

The number flux $N$ is calculated by sampling the molecules impinging on the surface by unit time and unit area. Results are normalized by $n_{\infty} V_{\infty}$, where $n_{\infty}$ is the freestream number density and $V_{\infty}$ is the freestream velocity.

The dependence of the dimensionless number flux to variations on the leading edge nose thickness is depicted in Figs. 3a to 3d for power law exponents of $2 / 3,0.7,3 / 4$ and 0.8 , respectively, as a function of the dimensionless arc length $s / \lambda_{\infty}$. Also, the dimensionless number flux for the circular cylinder case and that predicted by assuming free molecular (FM) flow ${ }^{17}$ are included for reference in this set of plots. It is seen from these figures that the dimensionless number flux to the front surface relies on the nose thickness in that it increases with increasing the thickness $t$. One possible reason for this behavior may be related to the collisions of two groups of molecules; the molecules reflecting from the nose of the leading edge and the molecules oncoming from the freestream. The molecules that are reflected from the body surface, which have a lower kinetic energy interact with the oncoming freestream molecules, which have a higher kinetic energy. Thus, the surface-reflected molecules re-collide with the body surface, which produce an increase in the dimensionless number flux in this region.

For a fixed power law exponent, it is also observed from these figures that the dimensionless number flux is high near the stagnation point and slightly decreases along the front surface up to the flat-face/afterbody junction with increasing the nose thickness (decreasing $K n_{t}$ ). After that, it drops off sharply along the body surface. As the nose thickness decreases (increasing $K n_{t}$ ), the dimensionless number flux approaches that limit value found for the zero-thickness $\operatorname{case}^{4}\left(K n_{t}=\infty\right)$. As the power law exponent increases from $2 / 3$ to 0.8 , a reduction in the dimensionless number flux is observed for the nose thickness cases investigated. In addition, for the $K n_{t}=100$ case $\left(t / \lambda_{\infty}=0.01\right)$, the dimensionless number flux approaches the value $\left(N / n_{\infty} V_{\infty}=1\right)$ predicted by the free molecular flow equations ${ }^{17}$.

In general, the qualitative trend for the dimensionless number flux is as expected, approaching the limit value obtained by the free molecular flow equations as the nose thickness decreases and the power law exponent increases, i.e., as the leading edge becomes sharp, and approaching the circular cylinder behavior with increasing the nose thickness, i.e., as the leading edge becomes blunt.

\section{B. Heat Transfer Coefficient}

The heat flux $q_{w}$ to the body surface is calculated by the net energy flux of the molecules impinging on the surface. The net heat flux $q_{w}$ is related to the sum of the translational, rotational and vibrational energies of both incident and reflected molecules. A flux is regarded as positive if it is directed toward the body surface. The heat flux is normalized by $\rho_{\infty} V_{\infty}^{3} / 2$ and presented in terms of heat transfer coefficient $C_{h}$.

The heat flux $q_{w}$ to the body surface was defined in terms of the incident and reflected flow properties, and based upon the gas-surface interaction model of fully accommodated, complete diffuse re-emission. The diffuse model assumes that the molecules are reflected equally in all directions, quite independently of their incident speed and direction. Due to the diffuse reflection model, the reflected thermal velocity of the molecules impinging on the surface is obtained from a Maxwellian distribution that takes into account for the temperature of the body surface. In this fashion, as the wall temperature is the same for all the cases 

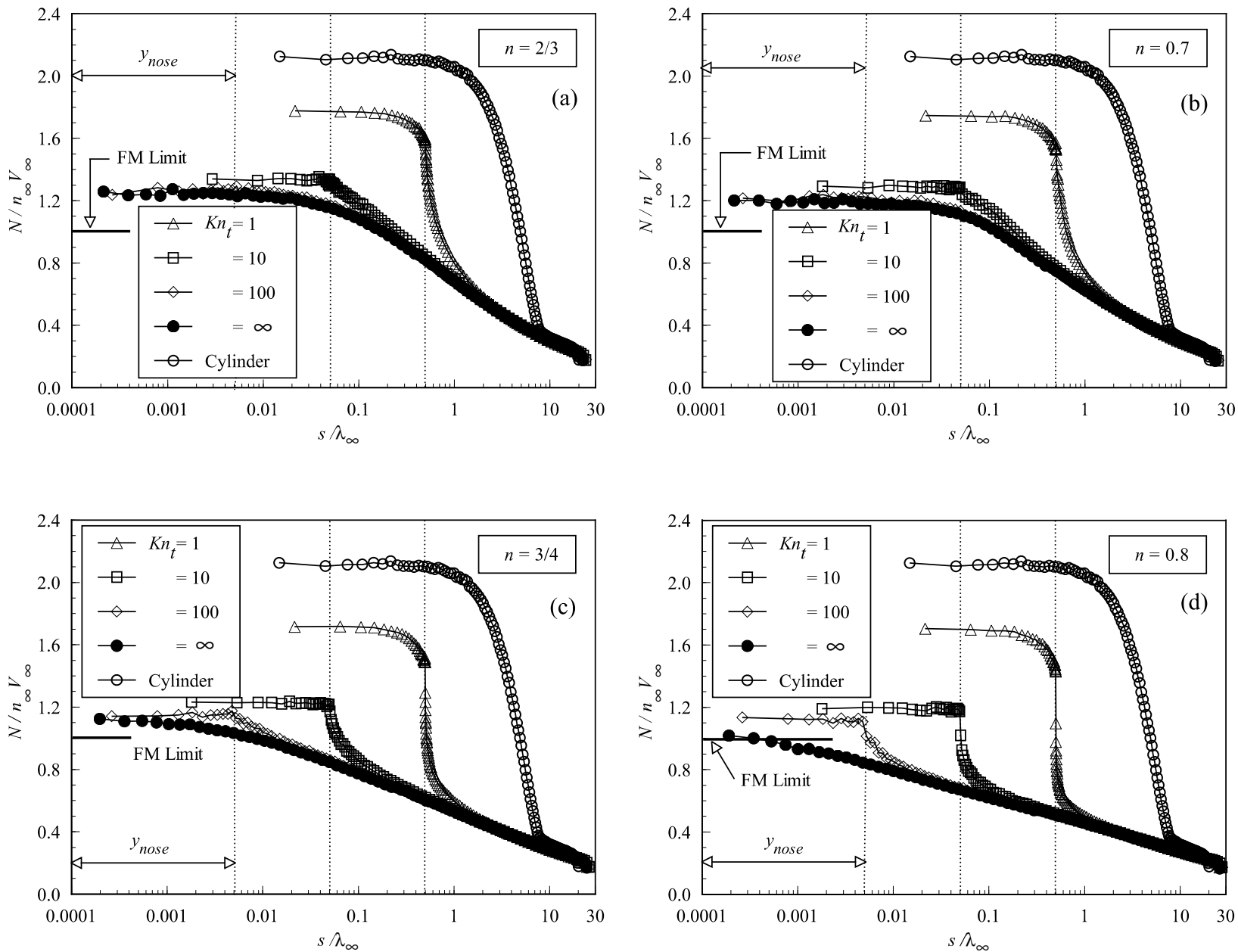

Figure 3. Dimensionless number flux $N / n_{\infty} V_{\infty}$ along the body surface as a function of the Knudsen number $K n_{t}$ for power-law afterbody exponents of (a) $2 / 3$, (b) 0.6 , (c) $3 / 4$ and (d) 0.8 .

investigated, the number of molecules impinging on the surface plays the important role on the reflected contribution to the net heat flux to the body surface.

The leading edge nose thickness effect on heat transfer coefficient $C_{h}$ is plotted in Figs. 4 a to $4 \mathrm{~d}$ for power law exponents of $2 / 3,0.7,3 / 4$ and 0.8 , respectively, as a function of the dimensionless distance $s / \lambda_{\infty}$ along the surface measured from the stagnation point. For comparison purpose, the heat transfer coefficient $C_{h}$ for the circular cylinder case is also shown as well as that predicted by free molecular flow. It is seen from these figures that the heat transfer coefficient $C_{h}$ is sensitive to the nose thickness. As would be expected, the flatter the leading edge is the lower the heat transfer coefficient at the stagnation point. Also, the heat transfer coefficient remains essentially constant over the first half of the front surface, but then increases in the vicinity of the flat-face/afterbody junction for the flattest case investigated, $K n_{t}=1\left(t / \lambda_{\infty}=1\right)$. Subsequently, the heat transfer coefficient decreases sharply and continues to decline along the body surface.

Usually, the stagnation region is generally considered as being the most thermally stressed zone in sharp/blunt bodies, as shown by the power law cases investigated defined by $K n_{t}=\infty\left(t / \lambda_{\infty}=0\right)$. Nevertheless, as a flat nose is introduced in these power law shapes, the most severe heat transfer region moves to 

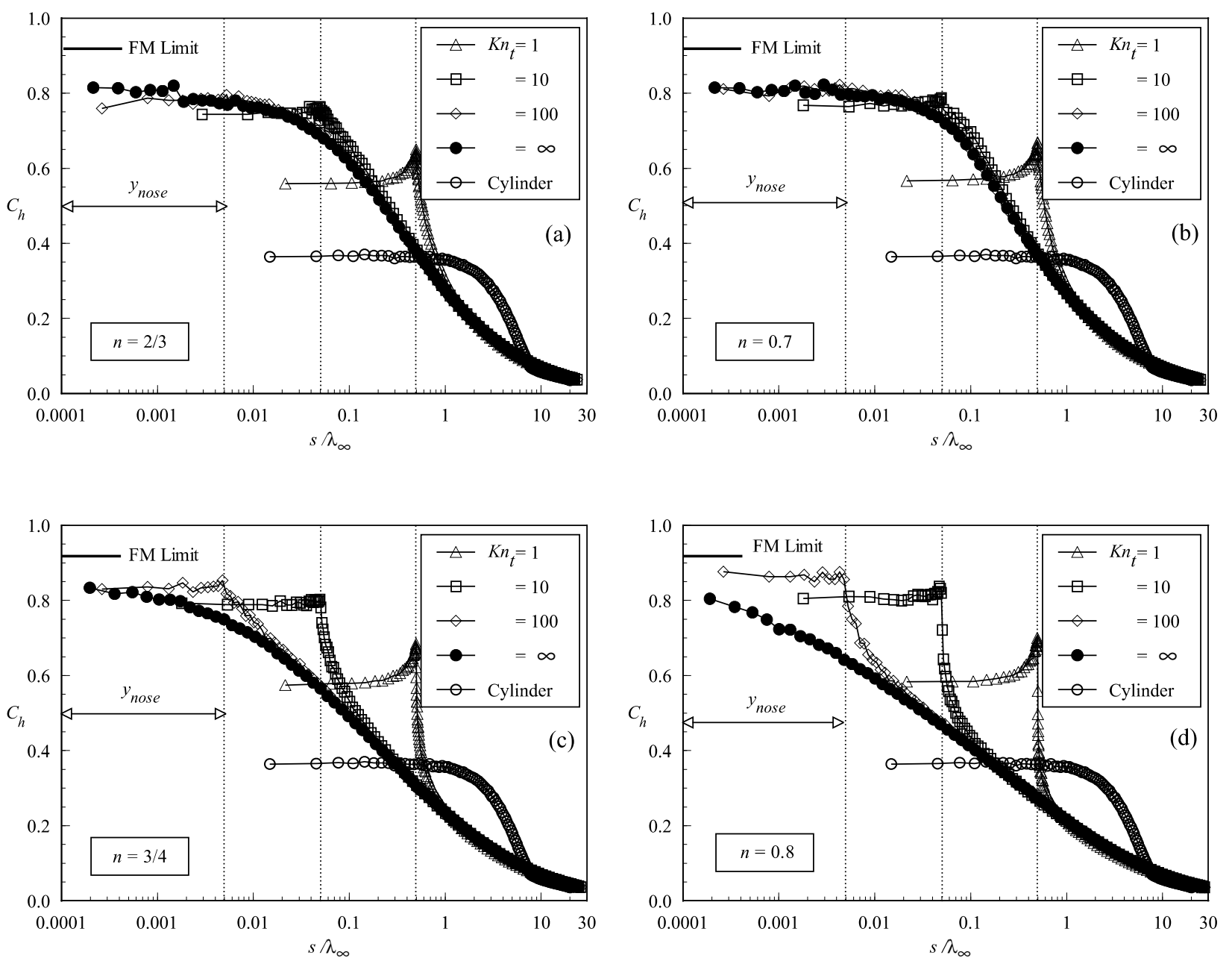

Figure 4. Heat transfer coefficient $C_{h}$ along the body surface as a function of the Knudsen number $K n_{t}$ for power-law afterbody exponents of (a) $2 / 3$, (b) 0.6 , (c) $3 / 4$ and (d) 0.8 .

the flat-face/afterbody junction. As the number of molecules impinging on the front surface decreases in the vicinity of the flat-face/afterbody junction (see Fig. 3), then the velocity of the molecules increases as the flow approaches the junction of the leading edge in order to increase the heat transfer coefficient. Moreover, the contribution of the translational energy to the net heat flux varies with the square of the thermal velocity of the molecules.

The heat transfer coefficient at the stagnation point predicted by the free molecular equations ${ }^{17}$ is $C_{h o}=$ 0.912, as shown in Figs. 4a to 4d. According to these figures, the stagnation point heating tends to this limit value as the leading edge becomes sharp, i.e., by reducing the nose thickness of the leading edges (increasing $K n_{t}$ ) and by increasing the power law exponent $n$.

In contrast to the power law bodies, the heat transfer coefficient $C_{h}$ for the circular cylinder remains essentially constant over the first half of the cylindrically portion of the leading edge, but then decreases sharply up to the cylinder/wedge junction. In addition, the heat transfer coefficient over the circular cylinder varies by an order of magnitude from the stagnation point to the cylinder/wedge junction. By considering that the heat transfer coefficient at the stagnation point $C_{h o}$ as well as $C_{h}$ is inversely proportional to $\sqrt{R}$ for 
the circular cylinder case, then decreasing the nose radius increases the value of $d C_{h} / d s$ by the factor $1 / R^{3 / 2}$. Consequently, for small nose radius $d C_{h} / d s$ is very large and, therefore, conduction plays an important role.

At this point, it seems important to compare the heat transfer coefficient at the stagnation point of the flat-nose power-law shapes $\left(C_{h o}\right)_{p w r}$ with that for the circular cylinder $\left(C_{h o}\right)_{c y l}$ that generated the power law shapes. Table 3 displays the ratio of $\left(C_{h o}\right)_{p w r}$ to $\left(C_{h o}\right)_{c y l}=0.366$ for the cases investigated as a function of the Knudsen number $K n_{t}$. According to Table 3, a substantial reduction in the heat transfer coefficient at the stagnation point is obtained by introducing the flat nose in the power law shapes. As a reference, for power law exponent of $2 / 3,\left(C_{h o}\right)_{p w r}$ reduces from $114.5 \%$ to $52.7 \%$, compared to $\left(C_{h o}\right)_{c y l}$, when the dimensionless nose thickness $t / \lambda_{\infty}$ increases from 0 to 1 . It is also noted that a similar behavior is obtained to power law exponent of 0.8 , changing from $140.2 \%$ to $59.6 \%$ for the same variation in the nose thickness.

Table 3. Heat transfer coefficient comparison at the stagnation point, $\left(C_{h o}\right)_{p w r} /\left(C_{h o}\right)_{c y l}$.

\begin{tabular}{ccccc}
\hline \hline Exp. $n$ & $K n_{t}=\infty$ & $K n_{t}=100$ & $K n_{t}=10$ & $K n_{t}=1$ \\
\hline $2 / 3$ & 2.145 & 2.123 & 2.033 & 1.527 \\
0.6 & 2.243 & 2.235 & 2.098 & 1.549 \\
$3 / 4$ & 2.344 & 2.273 & 2.156 & 1.574 \\
0.8 & 2.402 & 2.374 & 2.205 & 1.596 \\
\hline \hline
\end{tabular}

Referring to Table 3, it is also observed a considerable reduction in the heat transfer coefficients of the new power law shapes when they are compared to the corresponding zero-thickness cases. For the zero-thickness cases $\left(K n_{t}=\infty\right)$, the heat transfer coefficient increased by around $12 \%$ as the power law exponent increased from $2 / 3$ to 0.8 . In contrast, for the $t / \lambda_{\infty}=1$ case $\left(K n_{t}=1\right)$, the heat transfer coefficient increased only $4.5 \%$ as the power law exponent increased from $2 / 3$ to 0.8 .

\section{Pressure Coefficient}

The pressure $p_{w}$ on the body surface is calculated by the sum of the normal momentum fluxes of both incident and reflected molecules at each time step. Results are presented in terms of the pressure coefficient $C_{p}$.

The variation of the pressure coefficient $C_{p}$ caused by changes in the leading edge nose thickness is demonstrated in Figs. 5a to 5 d for power law exponents of $2 / 3,0.7,3 / 4$ and 0.8 , respectively. It can be noted from these figures that the pressure coefficient is high along the front surface, basically a constant value along it, and decreases dramatically along the afterbory surface in the vicinity of the flat-face/afterbody junction. This effect is more pronounced with decreasing Knudsen number $K n_{t}$, i.e., as the nose becomes flatter.

Plotted along with the computational solution for pressure coefficient is the pressure coefficient predicted by the free molecular flow equations and that for the circular cylinder. For the circular cylinder case, the pressure coefficient $C_{p}$ follows the same trend presented by the heat transfer coefficient in that it remains constant over the first half of the cylindrically portion of the leading edge, but then decreases sharply up to the cylinder/wedge junction. Also, the pressure coefficient $C_{p}$ varies by one order of magnitude from the stagnation point to the cylinder/wedge junction.

The pressure coefficient $C_{p}$ predicted by the free molecular flow equations on the front surface is 2.35 . Therefore, for the thinnest flat leading edge investigated, $t / \lambda_{\infty}=0.01$, which corresponds to $K n_{t}=100$, the flow seems to approach the free collision flow in the vicinity of the stagnation point (Fig. 5d), as was pointed out earlier. 

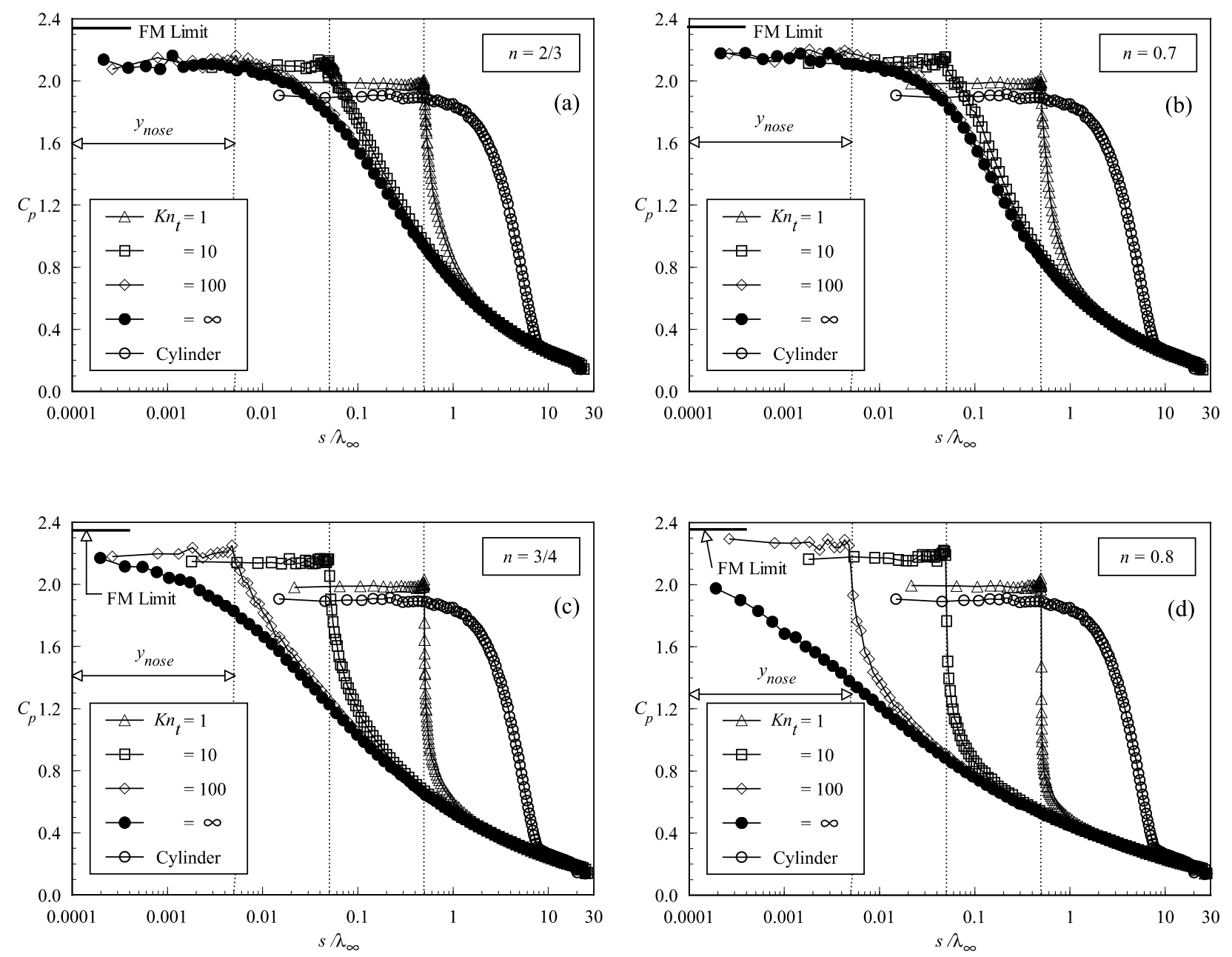

Figure 5. Pressure coefficient $C_{p}$ along the body surface as a function of the Knudsen number $K n_{t}$ for power-law afterbody exponents of (a) $2 / 3$, (b) 0.6, (c) $3 / 4$ and (d) 0.8 .

\section{Skin Friction Coefficient}

The shear stress $\tau_{w}$ on the body surface is calculated by averaging the tangential momentum transfer of the molecules impinging on the surface. For the diffuse reflection model imposed for the gas-surface interaction, reflected molecules have a tangential moment equal to zero, since the molecules essentially lose, on average, their tangential velocity component.

The shear stress $\tau_{w}$ on the body surface is normalized by $\rho_{\infty} V_{\infty}^{2} / 2$ and presented in terms of the dimensionless skin friction coefficient $C_{f}$. The influence of the leading edge nose thickness on the skin friction coefficient obtained by DSMC method is displayed in Figs. 6a to $6 \mathrm{~d}$ for power law exponents of 2/3, 0.7, 3/4 and 0.8 , respectively, and parameterized by the thickness Knudsen number. According to these figures, the skin friction coefficient $C_{f}$ is zero at the stagnation point and slightly increases along the front surface up to the flat-face/afterbody junction of the leading edge. After that, $C_{f}$ increases meaningfully to a maximum value that depends on the nose thickness, and decreases downstream along the body surface by approaching the skin friction coefficient predicted by the reference case of zero thickness, $K n_{t}=\infty$. In addition, smaller 

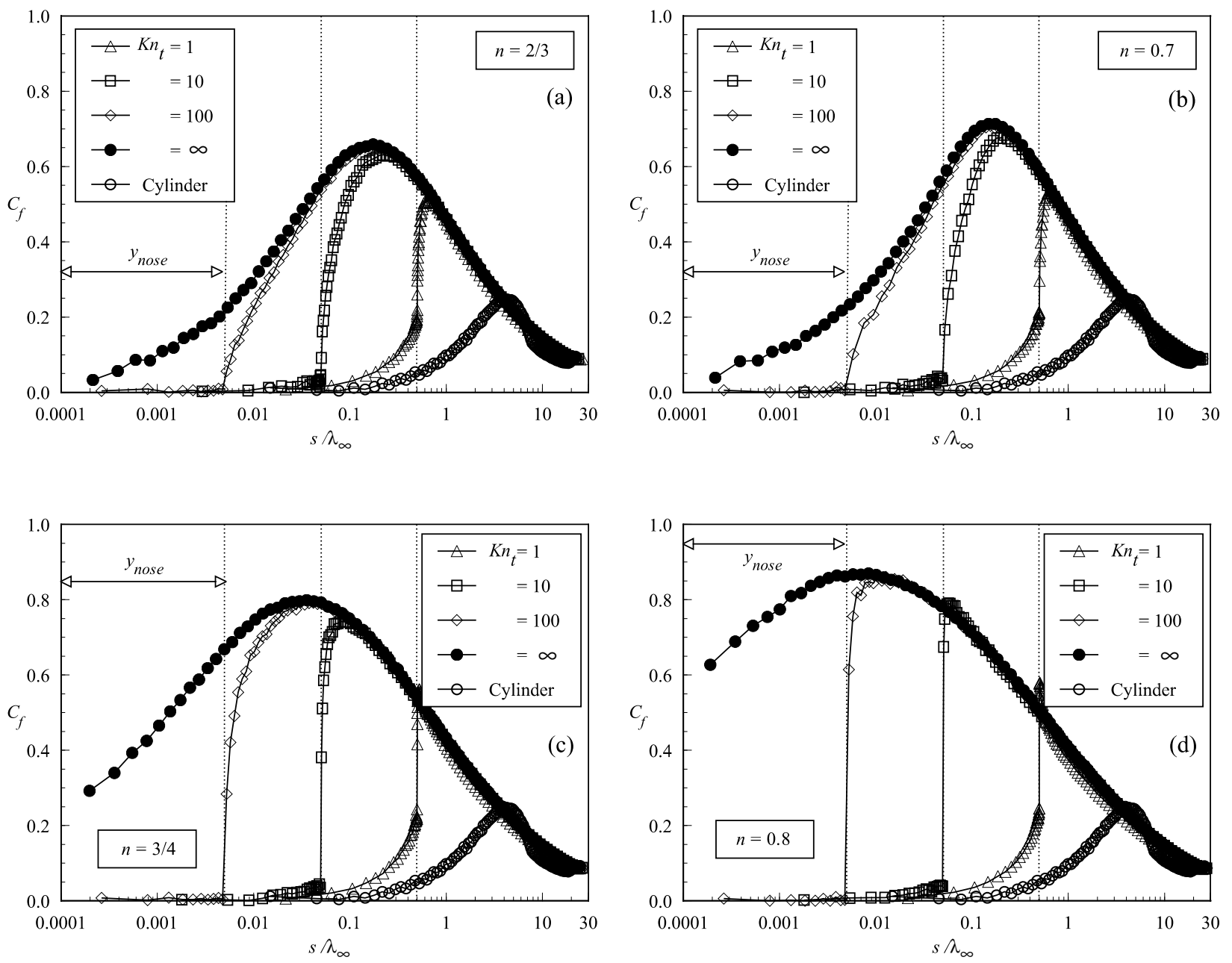

Figure 6. Skin friction coefficient $C_{f}$ along the body surface as a function of the Knudsen number $K n_{t}$ for power-law afterbody exponents of (a) $2 / 3$, (b) 0.6 , (c) $3 / 4$ and (d) 0.8 .

thickness $t$ (larger $K n_{t}$ ) leads to higher peak value for the skin friction coefficient $C_{f}$. Also, smaller thickness $t$ displaces the peak value to near the flat-face/afterbody junction.

The skin friction coefficient $C_{f}$ predicted by the free molecular flow equations ${ }^{17}$ is zero along the frontal surface of the bodies and exhibits its maximum value on the afterbody surface at a station that corresponds to a body slope of 45 degree (not shown). Similarly, the maximum values of $C_{f}$ for the nose thicknesses investigated occur very close to the same station. The number of molecules by unit time and unit area impinging on the body is high on the front surface of the leading edge and low on the afterbody portion of the body (see Fig. 3). In contrast, the tangential component of the molecular velocity is basically zero on the front surface and high along the afterbody surface (due to the flow expansion), where the velocities of the molecules are those that are more characteristic of the freestream velocity. As a result, the product of both properties, which is proportional to the shear stress (skin friction coefficient), presents the maximum value around station of 45 degree. Furthermore, attention should be paid to the fact that a body slope of 45 degree corresponds to a different arc length $s / \lambda_{\infty}$ for the nose thicknesses investigated. Also, as the power 
law exponent $n$ increases the arc length $s / \lambda_{\infty}$ corresponding to a slope of 45 degree approaches the shoulder of the shapes.

\section{E. Total Drag Coefficient}

The drag on a surface in a gas flow results from the interchange of momentum between the surface and the molecules colliding with the surface. The total drag is obtained by the integration of the pressure $p_{w}$ and shear stress $\tau_{w}$ distributions from the stagnation point of the leading edge to the station $L$ that corresponds to the tangent point common to all the leading edges (see Fig. 1). It is important to mention that the values for the total drag were obtained by assuming the shapes acting as leading edges. Therefore, no base pressure effects were taken into account on the calculations. Results for total drag are normalized by $\rho_{\infty} V_{\infty}^{2} H / 2$ and presented as total drag coefficient $C_{d}$ and its components of pressure drag coefficient $C_{p d}$ and the skin friction drag coefficient $C_{f d}$.

The extent of the changes in the total drag coefficient $C_{d}$ with the leading edge nose thickness is demonstrated in Fig. 7a to $7 \mathrm{~d}$ for power law exponents of $2 / 3,0.7,3 / 4$ and 0.8 , respectively, along with the drag coefficient for the circular cylinder case. It is seen that as the leading edge becomes flatter the contribution of the pressure drag $C_{p d}$ to the total drag increases and the contribution of the skin friction drag $C_{f d}$ decreases. As the net effect on total drag coefficient $C_{d}$ depends on these to opposite behaviors, hence no appreciable changes are observed in the total drag coefficient for the nose thicknesses investigated.

Referring to Figs. 7a to $7 \mathrm{~d}$, it can be seen that the total drag coefficient $C_{d}$ increased slightly by a rise in the nose thickness $t$. The major contribution to the total drag coefficient is attributed to the skin friction coefficient, which decreases with increasing the nose thickness $t$. In contrast, for the circular cylinder case, the major contribution to the total drag coefficient is attributed to the pressure, that accounts for $89 \%$ of the total drag.

At this point, it seems important to compare the total drag coefficient of the flat-nose power-law shapes $\left(C_{d}\right)_{\text {pwr }}$ with that for the circular cylinder $\left(C_{d}\right)_{c y l}$ that generated the power law shapes. Table 4 illustrates the ratio of $\left(C_{d}\right)_{p w r}$ to $\left(C_{d}\right)_{c y l}=1.519$ for the cases investigated as a function of the Knudsen number $K n_{t}$. According to Table 4, appreciable changes is observed in the total drag coefficient by introducing the flat nose in the power law shapes. As a reference, for power law exponent of $2 / 3,\left(C_{d}\right)_{p w r}$ increases from $71.0 \%$ to only $72.3 \%$, compared to $\left(C_{d}\right)_{c y l}$, when the dimensionless nose thickness $t / \lambda_{\infty}$ increases from 0 to 1 . It is also noted that a similar behavior is obtained to power law exponent of 0.8 , changing from $67.6 \%$ to $68.5 \%$ for the same variation in the nose thickness.

Table 4. Comparison of the total drag coefficient for flat-nose power-law shapes with that for the circular cylinder, $\left(C_{d}\right)_{p w r} /\left(C_{d}\right)_{c y l}$.

\begin{tabular}{ccccc}
\hline \hline Exp. $n$ & $K n_{t}=\infty$ & $K n_{t}=100$ & $K n_{t}=10$ & $K n_{t}=1$ \\
\hline $2 / 3$ & 0.710 & 0.711 & 0.711 & 0.723 \\
0.6 & 0.716 & 0.716 & 0.716 & 0.724 \\
$3 / 4$ & 0.687 & 0.688 & 0.688 & 0.697 \\
0.8 & 0.676 & 0.677 & 0.677 & 0.685 \\
\hline \hline
\end{tabular}

\section{Concluding Remarks}

The computations of a rarefied hypersonic flow on blunt bodies have been performed by using the Direct Simulation Monte Carlo method. The calculations provided information concerning the nature of the aerodynamic surface quantities for a family of contours composed by a flat nose followed by a curved afterbody 

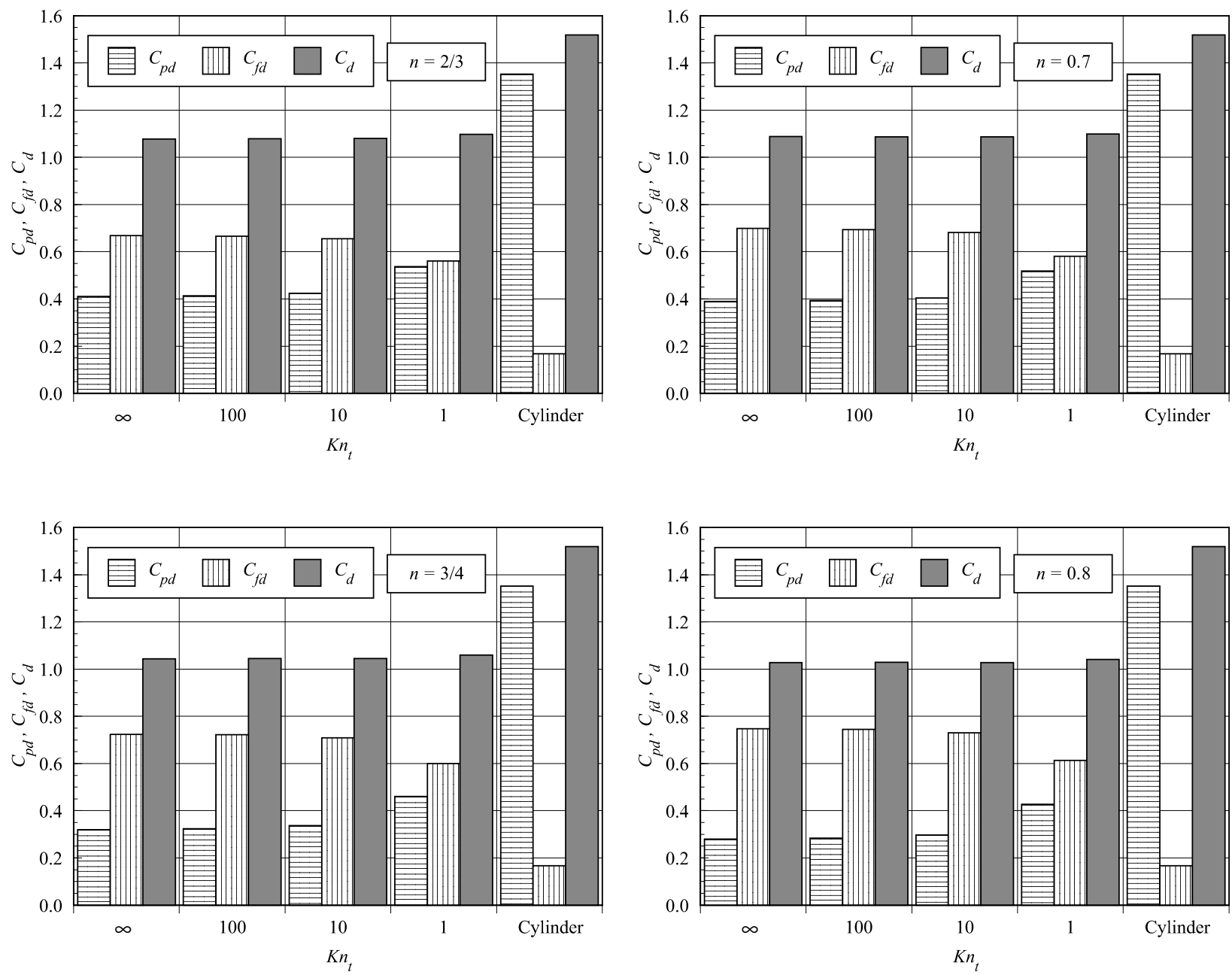

Figure 7. Pressure drag $C_{p d}$, skin friction drag $C_{f d}$ and total drag $C_{d}$ coefficients as a function of the Knudsen number $K n_{t}$ for power-law afterbody exponents of (a) 2/3, (b) 0.6, (c) $3 / 4$ and (d) 0.8 .

surface defined by power-law shapes.

The aerodynamic performance of these blunt shapes was compared to a corresponding circular cylinder leading edge shape, typically used in blunting sharp leading edges for heat transfer considerations. It was found that the stagnation point heating is still higher and the total drag is lower on the new blunt shapes than the representative circular cylinder solution in this geometric comparison. Thus, in general, these shapes behave as if they had a sharper profile than their representative circular cylinder. However, these shapes have more volume than the circular cylinder geometry. Hence, although stagnation point heating on these new shapes may be higher as compared to the circular cylinder, the overall heat transfer to these leading edges may be tolerate if there is an active cooling because additional coolant may be placed in the leading edge. Moreover, shock standoff distance on a cylinder scales with the radius of curvature, therefore cylindrical bluntness added for heating rate reduction will also tend to displace the shock wave, allowing pressure leakage. In this context, as the new shapes behave as if they were sharper profiles than the circular cylinder, they may display smaller shock detachment distances than the corresponding circular cylinder. Nevertheless, the shock wave structure on these new shapes is the subject for future work. 


\section{Acknowledgments}

The financial support provided by Fundação de Amparo à Pesquisa do Estado de São Paulo (grant FAPESP 2003/01765-9) is gratefully acknowledged.

\section{References}

${ }^{1}$ Mason, W. H. and Lee, J., "Aerodynamically Blunt and Sharp Bodies," Journal of Spacecraft and Rockets, Vol. 31, No. 3, 1994, pp. 378-382.

${ }^{2}$ O'Brien, T. F. and Lewis, M. J., "Power Law Shapes for Leading-Edge Blunting with Minimal Shock Standoff," Journal of Spacecraft and Rockets, Vol. 36, No. 5, 1999, pp. 653-658.

${ }^{3}$ Santos, W. F. N., "Direct Simulation Monte Carlo of Rarefied Hypersonic Flow on Power Law Shaped Leading Edges," Ph.D. Dissertation, Dept. of Aerospace Engineering, University of Maryland, College Park, MD, Dec., 2001.

${ }^{4}$ Santos, W. F. N., and Lewis, M. J., "Power Law Shaped Leading Edges in Rarefied Hypersonic Flow," Journal of Spacecraft and Rockets, Vol. 39, No. 6, 2002, pp. 917-925.

${ }^{5}$ Santos, W. F. N., and Lewis, M. J., "Angle of Attack Effect on Rarefied Hypersonic Flow over Power Law Shaped Leading Edges," in 23rd International Symposium on Rarefied Gas Dynamics, Whistler, BC, Canada, July 20-25, 2002.

${ }^{6}$ Santos, W. F. N., and Lewis, M. J., "Shock Wave Structure in a Rarefied Hypersonic Flow on Power Law Shaped Leading Edges," in 41st AIAA Aerospace Sciences Meeting and Exhibit, AIAA Paper 2003-1134, Reno, NV, January 6-9, 2003.

${ }^{7}$ Santos, W. F. N., and Lewis, M. J., "Aerodynamic Heating Performance of Power Law Leading Edges in Rarefied Hypersonic Flow," in 36th AIAA Thermophysics Conference, AIAA Paper 2003-3894, Orlando, FL, June 23-26, 2003.

${ }^{8}$ Santos, W. F. N., and Lewis, M. J., "Effects of Compressibility on Rarefied Hypersonic Flow over Power Law Leading Edges," in 42nd AIAA Aerospace Sciences Meeting and Exhibit, AIAA Paper 2004-1181, Reno, NV, January 5-8, 2004.

${ }^{9}$ Santos, W. F. N., and Lewis, M. J., "Calculation of Shock Wave Structure over Power Law Bodies in Hypersonic Flow," Journal of Spacecraft and Rockets, To appear, in press.

${ }^{10}$ Nonweiler, T. R. F., "Aerodynamic Problems of Manned Space Vehicles," Journal of the Royal Aeronautical Society, Vol. 63, Sept, 1959, pp. 521-528.

${ }^{11}$ Anderson, J. L., "Tethered Aerothermodynamic Research for Hypersonic Waveriders," in Proceedings of the 1st International Hypersonic Waverider Symposium, Univ. of Maryland, College Park, MD, 1990.

${ }^{12}$ Potter, J. L. and Rockaway, J. K., "Aerodynamic Optimization for Hypersonic Flight at Very High Altitudes," in Rarefied gas Dynamics: Space Science and Engineering, edited by B. D. Shizgal and D. P. Weaver, Vol. 160, Progress in Astronautics and Aeronautics, AIAA New York, 1994, pp.296-307.

${ }^{13}$ Rault, D. F. G., "Aerodynamic Characteristics of a Hypersonic Viscous Optimized Waverider at High Altitude," Journal of Spacecraft and Rockets, Vol. 31, No. 5, 1994, pp. 719-727.

${ }^{14}$ Graves, R. E. and Argrow, B. M., "Aerodynamic Performance of an Osculating-Cones Waverider at High Altitudes," in 35th AIAA Thermophysics Conference, AIAA Paper 2001-2960, Anaheim, CA, 2001.

${ }^{15}$ Reller Jr., J. O., 1957, "Heat Transfer to Blunt Nose Shapes with Laminar Boundary Layers at High Supersonic Speeds," NACA RM-A57FO3a, 1957.

${ }^{16}$ Schaaf, S. A. and Chambre, P. L., Flow of Rarefied Gases, Princeton Aeronautical Paperbacks, Princeton University Press, Princeton, NJ, 1961.

${ }^{17}$ Bird, G. A., Molecular Gas Dynamics and the Direct Simulation of Gas Flows, Oxford University Press, Oxford, England, UK, 1994.

${ }^{18}$ Bird, G. A., "Monte Carlo Simulation in an Engineering Context," in Progress in Astronautics and Aeronautics: Rarefied gas Dynamics, edited by Sam S. Fisher, Vol. 74, part I, AIAA New York, 1981, pp. 239-255.

${ }^{19}$ Bird, G. A., "Perception of Numerical Method in Rarefied Gasdynamics, in Rarefied gas Dynamics: Theoretical and Computational Techniques," edited by E. P. Muntz, and D. P. Weaver and D. H. Capbell, Vol. 118, Progress in Astronautics and Aeronautics, AIAA, New York, 1989, pp. 374-395.

${ }^{20}$ Garcia, A. L., and, Wagner, W., "Time Step Truncation Error in Direct Simulation Monte Carlo," Physics of Fluids, Vol. 12, No. 10, 2000, pp. 2621-2633.

${ }^{21}$ Hadjiconstantinou, N. G., "Analysis of Discretization in the Direct Simulation Monte Carlo," Physics of Fluids, Vol. 12, No. 10, 2000, pp. 2634-2638.

${ }^{22}$ Borgnakke, C. and Larsen, P. S., "Statistical Collision Model for Monte Carlo Simulation of Polyatomic Gas Mixture," Journal of computational Physics, Vol. 18, No. 4, 1975, pp. 405-420.

${ }^{23}$ Alexander, F. J., Garcia, A. L., and, Alder, B. J., "Cell Size Dependence of Transport Coefficient in Stochastic Particle Algorithms," Physics of Fluids, Vol. 10, No. 6, 1998, pp. 1540-1542.

${ }^{24}$ Alexander, F. J., Garcia, A. L., and, Alder, B. J., "Erratum: Cell Size Dependence of Transport Coefficient is Stochastic Particle Algorithms," Physics of Fluids, Vol. 12, No. 3, 2000, pp. 731-731. 